\title{
EDITORIAL
}

\section{Nasal high-flow preoxygenation for endotracheal intubation in the critically ill patient? Maybe}

\author{
Gérald Chanques ${ }^{*}$ (D) and Samir Jaber
}

(C) 2019 Springer-Verlag GmbH Germany, part of Springer Nature

In this issue of Intensive Care Medicine, Guitton et al. [1] report the results of the PROTRACH study, a multicenter randomized controlled trial including 184 non-severely hypoxemic patients requiring intubation in the intensive care unit (ICU), which compared the lowest pulse oximetry (SpO2) throughout the intubation procedure (primary outcome), between two different preoxygenation methods: standard bag valve mask oxygenation (SMO) and high-flow nasal cannulae (HFNC). There was no significant difference for the primary outcome $(p=0.30)$ but several meaningful secondary outcomes were significantly in favor of HFNC, including a twofold lower rate of mild desaturations below $95 \%(12 \%$ vs. $23 \%, p=0.04)$ and three times fewer severe adverse events related to intubation ( $6 \%$ vs. $16 \%, p=0.03)$. The negative result for the primary outcome can be explained by the studied population. Eligible patients were non-severely hypoxemic or not hypoxemic at all, $72 \%$ of the entire cohort being intubated for coma. This explains why the primary outcome, the lowest $\mathrm{SpO} 2$ during intubation, was around $99-100 \%$ in both groups. Otherwise, the study was very well designed and conducted. The only concern is its unblinded nature. This is often the case for this kind of study except for the OPTINIV trial that used a blinding of the trial: an additional HFNC was used in the control group but without oxygen flow provided to the patient [2]. However, no design is perfect because HFNC might modify the intubation condition, as suggested by the PROTRACH [1] and PREOXYFLOW [3] studies: intubation was reported as being more frequently difficult

\footnotetext{
*Correspondence: g-chanques@chu-montpellier.fr Department of Anaesthesia and Intensive Care, Saint Eloi Montpellier University Hospital, and PhyMedExp, University of Montpellier, INSERM,
} CNRS, 80, avenue Augustin Fliche, 34295 Montpellier Cedex 5, France in the HFNC group in both studies. Anyway, analyses of primary and secondary outcomes were based on objective measurements.

More difficult to understand is that this new trial comes after a negative one published by the same group in 2015, the PREOXYFLOW study [3]. This multicenter study included $119 \mathrm{ICU}$ patients requiring intubation for acute respiratory failure (ARF) and showed no significant difference between standard mask preoxygenation and HFNC for primary and secondary outcomes related to the intubation procedure. Thus, PREOXYFLOW and PROTRACH studies seem to report distinct impacts on outcomes, the latter study being in favor of HFNC while no significant impact was reported in the former. How can the differences between these two studies be explained?

The two studies included patients at high risk of adverse events related to the intubation procedure in the ICU setting: ARF in the PREOXYFLOW study and coma (mostly) in the PROTRACH study are the two main risk factors of difficult intubation in ICU patients [4-6]. However, if these are the two main risk factors, the mechanisms leading to a difficult intubation are distinct. In case of ARF, the difficulty of intubation can be explained by a very short preparation time and perhaps by increased stress for physicians performing the procedure. In case of coma, oropharyngeal secretions are often increased, limiting the view of the glottis. In both PREOXYFLOW and PROTRACH studies, the time to prepare for intubation was sufficient, inherent to the study protocol and inclusion criteria, but difficulties in performing intubation in comatose patients in the latter study could explain the lower rate of adverse events in the HFNC group: this is because HFNC allowed for administering oxygen throughout the intubation procedure, which is called

\section{实


Table 1 Advantages of different preoxygenation techniques for tracheal intubation in the ICU setting

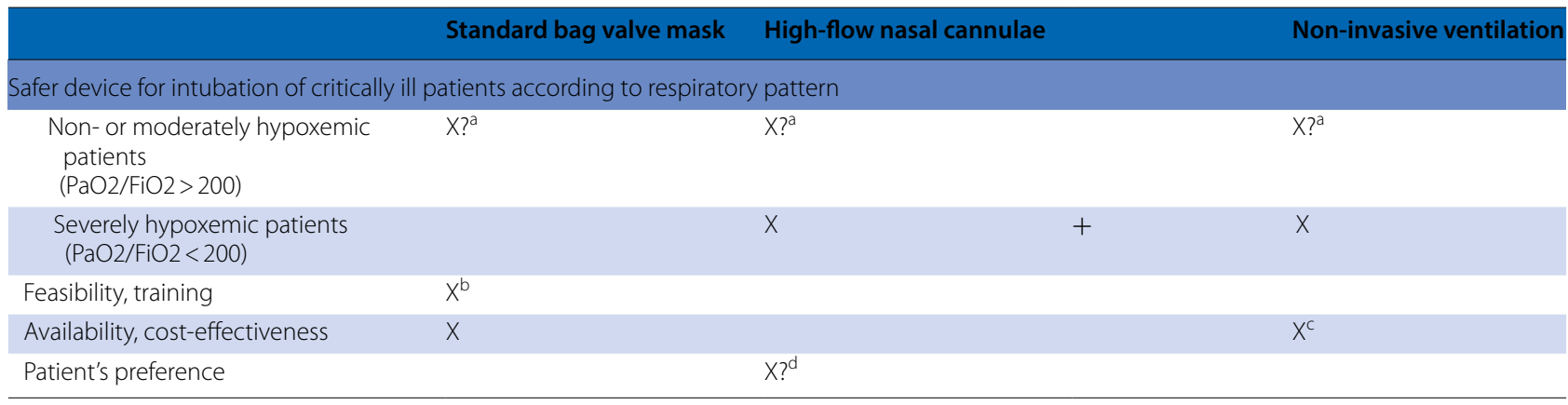

a High-flow nasal cannulae are safer than the recommended anesthetic/resuscitation bag-valve mask in the PROTRACH study [1]. However, possible confusion might be experienced by some investigators with the standard high FiO2 face mask used in the PREOXYFLOW study [3] (see text for details). Also, non-invasive ventilation provides the best oxygen reserves [14]

b Standard bag-valve mask might be the most feasible/easy to train device. This is because training seems necessary for intubation with HFNC: more frequent difficult intubation has been reported for intubation with this type of preoxygenation in two studies [1,3]. Moreover, NIV that can be used along with HFNC usually requires extensive training to be safe

c Cost-effectiveness is considered good for NIV because a mechanical ventilator is necessarily used after intubation in ICU, and because a simple anesthetic/ resuscitation face mask can be used for NIV instead of a dedicated NIV mask. In this situation, because NIV is provided for a very short time, air tightness between the mask and the patient's face can be ensured by the operator situated at the patient's head

$d$ In some studies, patient's preference has been reported to be better compared to face mask providing non-humidified oxygen, but this parameter has never been assessed for preoxygenation before tracheal intubation

apneic oxygenation $[2,7,8]$. Data reported in the PROTRACH study can support this hypothesis. Indeed, there were significantly fewer desaturations under $95 \%$ (and a trend towards fewer desaturations under $80 \%, p=0.06$ ) in the HFNC group for a similar rate of intubation issues that could have exposed the patients to longer apnea (i.e., there were more frequent esophageal intubations and aspirations in the SMO group but more frequent difficult intubations in the HFNC group). Considering the latter and because a higher rate of difficult intubations (even if not significant) had already been reported with HFNC in the PREOXYFLOW study, operators should be aware of some difficulties with the use of HFNC during intubation. These should be easily prevented by checking the possibilities to correctly position and move the patient's head and neck during the laryngoscopy and intubation procedure according to standard guidelines $[9,10]$.

Another important physiological difference between the two studied populations is the presence or absence of ARF. Many ARFs are associated with lung diseases, such as edema, acute lung injury, and atelectasis that are all reasons for decreased lung aeration and thus for lower oxygen diffusion. In patients with ARF, such as those included in the PREOXYFLOW study [3], HFNC alone is probably not sufficient to prevent desaturation, and the use of non-invasive ventilation (NIV) to provide pressure support ventilation demonstrated also some conflicting results when used alone $[11,12]$. However, when used in association with HFNC to provide apneic oxygenation throughout the intubation procedure, NIV is associated with a significantly lower rate of severe desaturation during intubation for ARF [2].
Last but not least, intubation by operators inexperienced in airway management can also be a major risk factor for failure [4]. In this way, the choice of the standard preoxygenation device is paramount. There could have been bias in the PROTRACH study because of misunderstanding. The authors stated in their introduction that preoxygenation with a high $\mathrm{FiO} 2$ facial mask (HFFM) is recommended to delay arterial desaturation during intubation apnea. HFFM was indeed used in the PREOXYFLOW study but an SMO was used in the PROTRACH study. However, the complete protocol available in the supplementary material reported the name of HFFM (even if SMO was described) and said moreover that "the choice of bag and mask will be left to the discretion of each investigating center (i.e. their usual device)." Because most of the participating ICUs had already participated in the PREOXYFLOW study, some physicians might have mixed up the two different devices according to their own practices. This could explain the study results, i.e., that preoxygenation was not effective in the control group in some patients, because tracheal $\mathrm{FiO} 2$ is only between $60 \%$ and $70 \%$ with HFFM at $15 \mathrm{~L} / \mathrm{min}$ of oxygen instead of $95 \%$ with HFNC $\geq 45 \mathrm{~L} / \mathrm{min}$ [13]. The choice of the device is inherent to the type of training. Experienced operators in airway management use a face mask with inflatable cushion to minimize air leaks between the mask and the patient's face, and a balloon to ensure a $100 \% \mathrm{FiO} 2$, a device similar to the SMO described in the PROTRACH protocol.

In all, rather than opposing all these techniques, we could use them in association, and remember that: 
1. HFNC, if available, could be used in all patients to provide apneic oxygenation throughout the intubation procedure.

2. NIV, a widespread technique in the ICU setting, provides the best oxygen reserves [14] and should be used in association with HFNC in the most hypoxemic patients, but also maybe in all critically ill patients because these patients have a high risk of intubation issues.

3. SMO could also be used (or probably NIV) to ventilate the patient during the procedure, which has appeared recently to be a safer technique than avoiding ventilation during the intubation of mixed hypoxemic/non-hypoxemic critically ill patients [15]. Ventilation should be performed early in case of desaturation anyway.

For NIV preoxygenation and systematic SMO/NIV ventilation, however, the benefit-to-risk ratio remains to be investigated in the non-hypoxemic patients if they have a high risk of gastric inhalation. Also, cost-effectiveness/feasibility and patient preference remain to be taken into account (Table 1).

\section{Funding}

Not applicable.

\section{Compliance with ethical standards}

\section{Conflicts of interest}

In contexts unrelated to the present work, Prof. Chanques has consulted for and received honorarium from Orion Pharma and Aspen Medical France. Prof. Jaber has consulted for and received honorarium from the following companies: Dräger, Xenios, Medtronic and Fisher Paykel.

\section{Ethical approval}

An approval by an ethics committee was not applicable.

\section{Publisher's Note}

Springer Nature remains neutral with regard to jurisdictional claims in published maps and institutional affiliations.

\section{Received: 4 March 2019 Accepted: 9 March 2019}

Published online: 19 March 2019

\section{References}

1. Guitton C, Ehrmann S, Volteau C, Colin G, Maamar A, Jean-Michel V, Mahe P, Landais M, Brule N, Bretonnière C, Zambon O, Vourc'h M (2019) Nasal high-flow preoxygenation for endotracheal intubation in the critically ill patient: a randomized clinical trial. Intensive Care Med. https://doi. org/10.1007/s00134-019-05529-w

2. Jaber S, Monnin M, Girard M, Conseil M, Cisse M, Carr J, Mahul M, Delay JM, Belafia F, Chanques G, Molinari N, De Jong A (2016) Apnoeic oxygenation via high-flow nasal cannula oxygen combined with non-invasive ventilation preoxygenation for intubation in hypoxaemic patients in the intensive care unit: the single-centre, blinded, randomised controlled OPTINIV trial. Intensive Care Med 42:1877-1887
3. Vourc'h M, Asfar P, Volteau C, Bachoumas K, Clavieras N, Egreteau PY, Asehnoune K, Mercat A, Reignier J, Jaber S, Prat G, Roquilly A, Brule N, Villers D, Bretonniere C, Guitton C (2015) High-flow nasal cannula oxygen during endotracheal intubation in hypoxemic patients: a randomized controlled clinical trial. Intensive Care Med 41:1538-1548

4. De Jong A, Molinari N, Terzi N, Mongardon N, Arnal JM, Guitton C, Allaouchiche B, Paugam-Burtz C, Constantin JM, Lefrant JY, Leone M, Papazian L, Asehnoune K, Maziers N, Azoulay E, Pradel G, Jung B, Jaber S, AzuRea Network for the Frida-Rea Study Group (2013) Early identification of patients at risk for difficult intubation in the intensive care unit: development and validation of the MACOCHA score in a multicenter cohort study. Am J Respir Crit Care Med 187:832-839

5. De Jong A, Jaber S (2018) Focus on ventilation management. Intensive Care Med 44:2254-2256

6. Jaber S, Bellani G, Blanch L, Demoule A, Esteban A, Gattinoni L, Guérin C, Hill N, Laffey J, Maggiore S, Mancebo J, Mayo P, Mosier J, Navalesi P. Quintel M, Vincent J, Marini J (2017) The intensive care medicine research agenda for airways, invasive and noninvasive mechanical ventilation. Intensive Care Med 43:1352-1365

7. Frumin MJ, Epstein RM, Cohen G (1959) Apneic oxygenation in man. Anesthesiology 20:789-798

8. Papazian L, Corley A, Hess D, Fraser J, Frat J, Guitton C, Jaber S, Maggiore S, Nava S, Rello J, Ricard J, Stephan F, Trisolini R, Azoulay E (2016) Use of high-flow nasal cannula oxygenation in ICU adults: a narrative review. Intensive Care Med 42:1336-1349

9. Quintard H, I'Her E, Pottecher J, Adnet F, Constantin JM, De Jong A, Diemunsch P, Fesseau R, Freynet A, Girault C, Guitton C, Hamonic Y, Maury E, Mekontso-Dessap A, Michel F, Nolent P, Perbet S, Prat G, Roquilly A, Tazarourte K, Terzi N, Thille AW, Alves M, Gayat E, Donetti L (2017) Intubation and extubation of the ICU patient. Anaesth Crit Care Pain Med 36:327-341

10. Quintard $H$, I'Her E, Pottecher J, Adnet F, Constantin JM, De Jong A, Diemunsch P, Fesseau R, Freynet A, Girault C, Guitton C, Hamonic Y, Maury E, Mekontso-Dessap A, Michel F, Nolent P, Perbet S, Prat G, Roquilly A, Tazarourte K, Terzi N, Thille AW, Alves M, Gayat E, Donetti L (2019) Experts' guidelines of intubation and extubation of the ICU patient of French Society of Anaesthesia and Intensive Care Medicine (SFAR) and Frenchspeaking Intensive Care Society (SRLF): in collaboration with the pediatric Association of French-speaking Anaesthetists and Intensivists (ADARPEF), French-speaking Group of Intensive Care and Paediatric Emergencies (GFRUP) and Intensive Care Physiotherapy Society (SKR). Ann Intensive Care 9:13

11. Baillard C, Fosse JP, Sebbane M, Chanques G, Vincent F, Courouble P, Cohen Y, Eledjam JJ, Adnet F, Jaber S (2006) Noninvasive ventilation improves preoxygenation before intubation of hypoxic patients. Am J Respir Crit Care Med 174:171-177

12. Baillard C, Prat $G$, Jung B, Futier E, Lefrant JY, Vincent F, Hamdi A, Vicaut $E_{\text {, }}$ Jaber S (2018) Effect of preoxygenation using non-invasive ventilation before intubation on subsequent organ failures in hypoxaemic patients: a randomised clinical trial. Br J Anaesth 120:361-367

13. Chanques G, Riboulet F, Molinari N, Carr J, Jung B, Prades A, Galia F, Futier E, Constantin JM, Jaber S (2013) Comparison of three high flow oxygen therapy delivery devices: a clinical physiological cross-over study. Minerva Anestesiol 79:1344-1355

14. Bailly A, Ricard J, Le Thuaut A, Helms J, Kamel T, Mercier E, Lemiale V, Colin G, Mira J, Clere-Jehl R, Messika J, Dequin P, Boulain T, Azoulay E, Champigneulle B, Reignier J, Lascarrou J, Research in Intensive Care and Sepsis Group (CRICS-TRIGGERSEP) (2019) Compared efficacy of four preoxygenation methods for intubation in the ICU: retrospective analysis of Mcgrath Mac videolaryngoscope versus Macintosh laryngoscope (MACMAN) trial data. Crit Care Med. https://doi.org/10.1097/CCM.0000000000003656

15. Casey J, Janz D, Russell D, Vonderhaar D, Joffe A, Dischert K, Brown R, Zouk A, Gulati S, Heideman B, Lester M, Toporek A, Bentov I, Self W, Rice T, Semler M, PreVent Investigators and the Pragmatic Critical Care Research Group (2019) Bag-mask ventilation during tracheal intubation of critically ill adults. NEJM 380:811-821 